\title{
6-Mercaptopurine Self-Assembled Monolayers on Gold (001)-hex: Revealing the Fate of Gold Adatoms
}

Pilar Carro, Kathrin Müller, Flavia Lobo Maza, Carolina Vericat, Ulrich Starke, Klaus Kern, Roberto C. Salvarezza, and Doris Grumelli

J. Phys. Chem. C, Just Accepted Manuscript • DOI: 10.1021/acs.jpcc.7b02732 • Publication Date (Web): 05 Apr 2017

Downloaded from http://pubs.acs.org on April 12, 2017

\section{Just Accepted}

"Just Accepted" manuscripts have been peer-reviewed and accepted for publication. They are posted online prior to technical editing, formatting for publication and author proofing. The American Chemical Society provides "Just Accepted" as a free service to the research community to expedite the dissemination of scientific material as soon as possible after acceptance. "Just Accepted" manuscripts appear in full in PDF format accompanied by an HTML abstract. "Just Accepted" manuscripts have been fully peer reviewed, but should not be considered the official version of record. They are accessible to all readers and citable by the Digital Object Identifier (DOI®). "Just Accepted" is an optional service offered to authors. Therefore, the "Just Accepted" Web site may not include all articles that will be published in the journal. After a manuscript is technically edited and formatted, it will be removed from the "Just Accepted" Web site and published as an ASAP article. Note that technical editing may introduce minor changes to the manuscript text and/or graphics which could affect content, and all legal disclaimers and ethical guidelines that apply to the journal pertain. ACS cannot be held responsible for errors or consequences arising from the use of information contained in these "Just Accepted" manuscripts. 


\section{6-Mercaptopurine Self-Assembled Monolayers on Gold (001)-hex: Revealing the Fate of Gold Adatoms}

Pilar Carro ${ }^{1}$, Kathrin Müller ${ }^{2}$, Flavia Lobo Maza ${ }^{3}$, Carolina Vericat $^{3}$, Ulrich Starke ${ }^{2}$, Klaus Kern ${ }^{2,4}$, Roberto C. Salvarezza ${ }^{3}$ and Doris Grumelli ${ }^{3 *}$

${ }^{1}$ Área de Química Física, Departamento de Química, Facultad de Ciencias, Universidad de La Laguna, Instituto de Materiales y Nanotecnología, Avda. Francisco Sánchez, s/n 38200-La Laguna, Tenerife, Spain

${ }^{2}$ Max Planck Institute for Solid State Research, Heisenbergstrasse 1, D-70569 Stuttgart, Germany

${ }^{3}$ Instituto de Investigaciones Fisicoquímicas Teóricas y Aplicadas (INIFTA), Facultad de Ciencias Exactas, Universidad Nacional de La Plata - CONICET-Sucursal 4 Casilla de Correo 16, (1900) La Plata, Argentina

4 Institut de Physique, Ecole Polytechnique Fédéerale de Lausanne, 1015 Lausanne, Switzerland

*Corresponding author: Doris Grumelli

email : doris@inifta.unlp.edu.ar 


\begin{abstract}
Thiol molecules adsorbed on gold have long ago become a model system for molecular self-assembly on metal substrates. In most cases the strong molecule-gold interaction is able to restructure the substrate, resulting in vacancy islands and steps. Today it is widely accepted that gold adatoms produced by this process form stable thiol-adatom complexes, usually termed "staples" (RS-Au $u_{a d}-S R$ or RS- $\left.\mathrm{Au}_{\mathrm{ad}}-\mathrm{SR}-\mathrm{Au}_{\mathrm{ad}}-\mathrm{SR}\right)$, which are the basic units of the self-assembled monolayers. Here we report on a different scenario for 6-mercaptopurine (6MP), a heterocyclic aromatic thiol, namely its adsorption on the $\mathrm{Au}(001)-(5 \times 20)$ reconstructed surface. Our results show that $6 \mathrm{MP}$ lifts the reconstruction upon adsorption, thus ejecting a large excess of gold adatoms. Surprisingly, 6MP molecules prefer to arrange in highly ordered adatom-free domains in the bridging configuration, while the ejected adatoms form gold islands. Our investigation reveals that the formation of thiol-gold adatom complexes is not always a thermodynamically favored process but rather depends on the nature of the thiol molecule.
\end{abstract}




\section{Introduction}

One of the key structural components in self-assembled monolayers (SAMs) of thiolates (RS) on $\mathrm{Au}$ surfaces are gold adatoms $\left(\mathrm{Au}_{\mathrm{ad}}\right)$, that form different thiol-adatom complexes commonly called staples (either RS-Au $u_{a d}-S R$ or RS-Au $u_{a d}-S R-A u_{a d}-S R$ species) ${ }^{1-2}$ which have been identified on thiol-protected Au nanoclusters (AuNC) $)^{3}$ and also on the surface of wellordered thiolate SAMs on $\mathrm{Au}(111) .^{4}$ In the former case, AuNC grow in the presence of $\mathrm{Au}(\mathrm{III})$ ions and thiolates, so that the staples could be formed as a product of the synthesis or by attack of the thiolates to the growing clusters resulting in gold atom removal from the surface. ${ }^{2,5}$ This last mechanism is evident during the self-assembly of alkanethiols on the $\mathrm{Au}(111)$ surface, where they induce the formation of either serrated steps or vacancy islands at terraces as a result of adatom ejection leading to staples formation. From the stoichiometry of the gold complexes, $0.16 \mathrm{Au}_{\mathrm{ad}} \mathrm{ML}$ are needed for the typical saturation coverage of thiols $\theta=0.33 \mathrm{ML}^{6}{ }^{6}$ In this case there is also a small contribution of 0.05 of $\mathrm{Au}_{\mathrm{ad}}$ arising from the lifting of the $\mathrm{Au}(111)-(22 \times \sqrt{3})$ reconstruction (the "herringbone" reconstruction) which is induced by thiol adsorption. It is now broadly accepted that staples are a general and thermodynamically favored binding motif of alkanethiolates on $\mathrm{Au}(111)$, a fact that has been confirmed by DFT calculations. ${ }^{1}$

In contrast, a general feature for the adsorption of small-sized aromatic thiols (with one or two aromatic rings) on $\mathrm{Au}(111)$ is that they lift the herringbone reconstruction and generate gold steps, but fail to produce vacancy islands, i.e. they are unable to extract Au atoms from terraces. In fact, rather than vacancy islands aromatic thiols induce the formation of small islands which have been assigned either to gold structures and/or to molecular aggregates. ${ }^{7-8}$ The failure of small aromatic thiols to form staple moieties has been explained considering their lower coverage and adsorption energy compared to alkanethiols (they do not compensate the energy cost to extract the $\mathrm{Au}$ atoms from the terraces). ${ }^{9}$ Similar arguments 
have been used to explain the absence of staples when 6-mercaptopurine (6MP, Figure 1), a small aromatic thiol of relevance for the treatment of some leukemias and autoimmune conditions because of its role as purine antagonist ${ }^{10}$, is adsorbed on the $\operatorname{Au}(111)^{7}$ and $\mathrm{Au}(001)-(1 \times 1)$ surfaces. ${ }^{11}$ However, there is also another possible reason for this behavior: a low chemical affinity of the aromatic molecules to the Au adatoms, a fact that could also explain the formation of Au islands rather than vacancy Au islands.

In this manuscript, we have placed 6MP molecules in a completely different scenario in order to test the molecule- $\mathrm{Au}_{\mathrm{ad}}$ reactivity on the substrate surface. 6MP was adsorbed on the $\mathrm{Au}(001)-(5 \times 20)$ (hereafter denoted as $\mathrm{Au}(001)-(\mathrm{hex}))$ surface, which exhibits a hexagonal surface reconstruction with a higher density of $\mathrm{Au}$ atoms in the first layer (0.25 ML of additional $\mathrm{Au}$ atoms). ${ }^{12}$ If the adsorption of molecules is able to lift the hex surface reconstruction then they would be in contact with a large amount of ejected Au adatoms $\left(\theta_{\mathrm{ad}}\right.$ $=0.25)$ whose fate would account for the affinity of the aromatic molecules to form the staples units. Our results from low-energy electron diffraction (LEED), scanning tunneling microscopy (STM), x-ray photoelectron spectroscopy (XPS), electrochemical measurements and density functional theory (DFT) calculations show that 6MP molecules indeed lift the hex reconstruction and chemisorb through a S-Au bond. However, they prefer to organize in wellordered staple-free lattices in a bridging configuration with gold adatoms forming small and irregular islands, i.e. this aromatic molecule is not prone to form staple moieties.

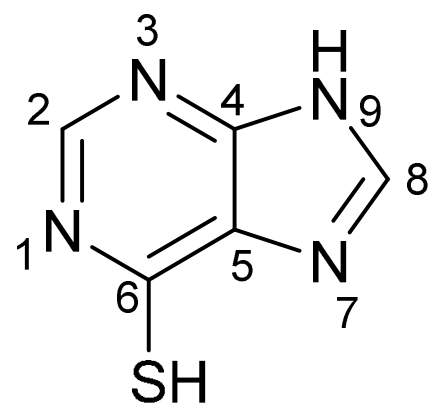

Figure1. Chemical structure of the 6-mercaptopurine (6MP) molecule 


\section{Experimental Methods}

\section{Sample preparation}

$\mathrm{Au}(001)$ single-crystal substrates (MaTeck) were cleaned by repeated cycles of sputtering with $\mathrm{Ar}^{+}$ions and annealing at $825 \mathrm{~K}$ under ultra-high vacuum (UHV) leading to the $\mathrm{Au}(001)$-(hex) surface reconstruction, ${ }^{12}$ which has been confirmed by LEED and STM. This surface is characterized by stripes $1.45 \mathrm{~nm}$ wide separated by $0.07 \mathrm{~nm}$ deep channels. SAMs of 6MP (Aldrich, 98\%) were prepared by immersion of the $\mathrm{Au}(001)$-hex surfaces in $100 \mu \mathrm{M}$ 6MP ethanolic solutions (BASF 99\%) for $30 \mathrm{~min}$. Then the samples were removed from the solution, rinsed with ethanol and dried under $\mathrm{N}_{2}$ before they were reintroduced into UHV.

\section{STM imaging}

Samples were imaged in a home-made UHV STM operating at room temperature. W tips were used with 100-600 mV tunneling voltages and 100-350 pA tunneling currents. The STM was calibrated in $\mathrm{x}, \mathrm{y}$ and $\mathrm{z}$ directions using the stripes of the well-known $\mathrm{Au}(001)-\mathrm{hex}$ surface reconstruction. WsXM software was used for image analysis. ${ }^{13}$

\section{Electrochemical experiments}

A home-built sample transfer system between UHV and electrochemical environment was used. After preparation or characterization the samples were brought to the transfer chamber and argon atmosphere (5.0 purity, $p=1$ bar) was established.

Cyclic voltammetry was made with an Autolab PGSTAT30 potentiostat and a three-electrode conventional electrochemical cell. A large area Pt coil was used as counter electrode and a silver/silver chloride $(3 \mathrm{M} \mathrm{KCl})$ electrode was employed as reference electrode $(\mathrm{RE})$. All the potentials in the text are referred to that RE. Aqueous $0.1 \mathrm{M} \mathrm{NaOH}$ solutions were prepared by using $\mathrm{NaOH}$ pellets (Sigma-Aldrich; $99.99 \%$ trace metals basis) and ultrapure water with 18.2 M $\Omega \mathrm{cm}$ resistivity (Millipore Products, Bedford). 
Thiol reductive electrodesorption curves were performed at room temperature by scanning the potential from $-0.3 \mathrm{~V}$ to $-1.4 \mathrm{~V}$ at $0.05 \mathrm{Vs}^{-1}$ in the $0.1 \mathrm{M} \mathrm{NaOH}$ solution. In each case the charge density (q) involved in the reductive desorption peak was obtained by integration of the peak area. The geometrical area of the single crystal electrode $\left(0.196 \mathrm{~cm}^{2}\right)$ was used to calculate the current densities. This figure was taken as an indication of the surface coverage of the thiol SAM.

\section{XPS and LEED measurements}

XPS and LEED measurements were performed in a separate UHV system. The freshly prepared 6MP SAMs were transferred into the UHV system. Photoemission experiments for 6MP SAMs were carried out in a commercial KRATOS AXIS ULTRA system with a monochromatized $\mathrm{Al} \mathrm{K \alpha}$ source with a base pressure in the lower $10^{-10}$ mbar range. The binding energy (BE) scales for the SAMs on Au surfaces were calibrated by setting the $\mathrm{Au}$ $4 \mathrm{f}_{7 / 2} \mathrm{BE}$ to $84.0 \mathrm{eV}$ with respect to the Fermi level. High-resolution S 2p, N 1s, C 1s and Au $4 \mathrm{f}$ spectra were acquired with an analyzer pass energy of $20 \mathrm{eV}$.

Analysis of the XPS data was performed with CasaXPS v 2.3.84 software. Shirley type backgrounds were subtracted from the spectra before the fitting procedure and peaks that are a combination of Lorenzian and Gaussian functions were employed for all regions. In the case of the S $2 p$ signal, the spin-orbit doublet separation was fixed at $1.18 \mathrm{eV}$ and an area ratio of 2:1 was used. The atomic concentrations were calculated by using calculated photoemission cross-sections according to Yeh and Lindau. ${ }^{14}$

LEED data were acquired in the same UHV system using a commercial SPECS ErLEED 1000-A. LEEDPAT software ${ }^{15}$ was used to simulate the obtained LEED patterns. The LEED of the clean $\mathrm{Au}(001)$-hex surface was obtained after cleaning the sample in the same UHV system and transferring it into the LEED chamber without breaking the vacuum.

\section{Computational Methods}


Density functional calculations were performed with the periodic plane-wave basis set code VASP 5.2.12. ${ }^{16-17}$ The scheme of non-local functional proposed by Dion et al, ${ }^{18} \mathrm{vdW}-\mathrm{DF}$, and the optimized Becke88 exchange functional optB88-vdW ${ }^{19}$ were used to take into account van der Waals (vdW) interactions. The projector augmented plane wave (PAW) method was used to represent the atomic cores using the PBE potential. ${ }^{20}$ The electronic wave functions were expanded in a plane-wave basis set with a $420 \mathrm{eV}$ cutoff energy. Optimal grids of Monkhorst-Pack ${ }^{21} \mathrm{k}$-points $2 \times 9 \times 1$ were used for numerical integration in the reciprocal space of the $(6 \times \sqrt{ } 2)$ unit cell used to simulate the $(3 \sqrt{ } 2 \times \sqrt{ } 2) \mathrm{R} 45^{\circ}$ surface structure described in the experimental results section. Because 6MP adsorption lifts the hex-reconstruction we have used a $\mathrm{Au}(001)-(1 \times 1)$ substrate represented by five atomic layers and a vacuum of $\sim 17 \AA$ that separates two successive slabs in our calculation. Surface relaxation is allowed in the three uppermost Au layers of the slab, as well as the atomic coordinates of the adsorbed species, were allowed to relax without further constraints. The atomic positions were relaxed until the force on the unconstrained atoms was $<0.03 \mathrm{eV}^{-1}$. The adsorbates were placed just on one side of the slab and all calculations include a dipole correction. The adsorbed 6MP species, taken as thiyl radicals ${ }^{22}$ were optimized in an asymmetric box of $20 \AA \times 20 \AA \times 40 \AA$. The calculated $\mathrm{Au}$ lattice constant is $4.16 \AA$, which compares reasonably well with the experimental value $(4.078 \AA){ }^{23}$ The average binding energy per adsorbed 6MP species on $\operatorname{Au}(001)-(1 \times 1)$ surfaces, $E_{b}$, is defined in Eq. [1]:

$$
E_{b}=\frac{1}{N_{\text {thiol }}}\left[E^{\text {thiol } / A u}-E^{A u}-N_{\text {thiol }} E^{\text {thiol }}\right]
$$

where, $E^{\text {thiol/Au }}, E^{A u}$ and $E^{\text {thiol }}$ stand for the total energy of the adsorbate-substrate system, the total energy of the Au slab, and the energy of the 6MP radical, respectively, whereas $N_{\text {thiol }}$ is the number of $6 \mathrm{MP}$ radicals in the surface unit cell. A negative number indicates that adsorption is exothermic with respect to the separate clean surface and 6MP radical. The 
Gibbs free energy of adsorption of each surface structure $(\gamma)$ was approximated through the total energy from DFT calculations by using equation [2]:

$$
\gamma=\frac{N_{\text {thiol }} E_{b}+E_{r e c}}{A}
$$

where $\mathrm{E}_{\mathrm{rec}}$ is the reconstruction energy per unit cell defined by,

$$
E_{\text {rec }}=E_{A u(100)}^{R}-E_{A u(100)}^{U}-n_{a d} E_{b u l k}^{A u}
$$

where $E_{A u(100)}^{R}$ and $E_{A u(100)}^{U}$ correspond to the energy of reconstructed $\mathrm{Au}(001)$ surface and unreconstructed $\mathrm{Au}(001)$ surface per unit cell, respectively; $E_{b u l k}^{A u}$ is the total energy of a bulk Au atom, $n_{a d}$ is the number of Au adatoms in the surface unit cell and $A$ is the unit cell area. Considering that we are concerned with free energy differences, it is reasonable to assume that the contributions coming from the configurational entropy, the vibrations and the work term $p V$, can be neglected. DFT calculations make systematic (but unknown) errors relative to the true solution of the Schrödinger equation because of the approximate nature of the exchange-correlation functional. However if two DFT calculations compare chemically similar states, then the systematic errors in these two calculations are also similar. In other words, relative energies can be calculated accurately. In this sense, we can therefore expect a good level of accuracy in the adsorption energy.

\section{Results and Discussion}

The LEED pattern of the clean $\mathrm{Au}(001)$ shown in Figure $2 \mathrm{a}$ is consistent with that already reported for the well-known $(5 \times 20)$ hex reconstruction. ${ }^{24}$ Clean surfaces were then used for the self-assembly of the 6MP molecules by immersion in the 6MP ethanolic solution for 30 $\min$. 


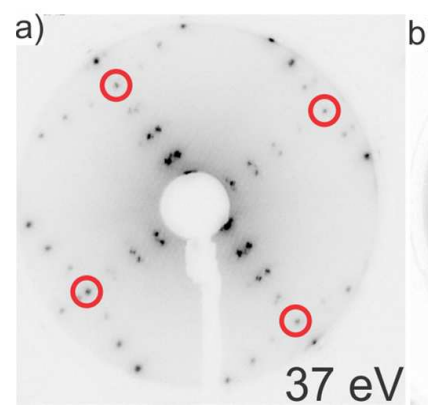

b)

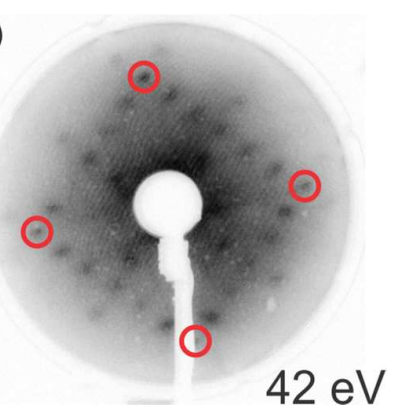

Figure 2. LEED patterns for (a) the clean Au(001)-hex surface and (b) the same substrate after a 30 min immersion in 6MP solution. Note that the LEED patterns are rotated with respect to each other due to different mounting orientations of the sample. The $(1,0)$ main spots are marked by red circles for easier comparison.

After immersion of the $\mathrm{Au}(001)$-hex substrate into the 6MP ethanolic solution a LEED pattern showing a $(3 \sqrt{ } 2 \times \sqrt{ } 2) \mathrm{R} 45^{\circ}$ reconstruction, commensurate with the $\mathrm{Au}(001)$ substrate, was obtained (Figure 2b). This gives a rectangular unit cell with unit cell parameters of $1.22 \times 0.41 \mathrm{~nm}^{2}$. The unit cell is rotated by $45^{\circ}$ with respect to the $<110>$ direction of the $\mathrm{Au}$ surface. Furthermore, one can notice that none of the spots in the LEED pattern coincides with those of the hex surface reconstruction (Figure 2a), evidencing that it has been lifted during the 6MP self-assembly. In contrast, immersion of the reconstructed substrate in pure ethanol for a similar time period does not affect the hex surface, i.e. ethanol adsorption is not able to lift the reconstruction (data not shown).

STM gives local information on how 6MP molecules organize on the Au substrate. The images show that most of the surface exhibits a large number of irregular islands 2-6 nm in size (Figure 3a) and height $h \approx 0.2 \mathrm{~nm}$ (Figure 3b). This value indicates that they are mostly $\mathrm{Au}$ islands resulting from the lifting of the hex reconstruction. The islands contain also an internal corrugation with $h \approx 0.03-0.05 \mathrm{~nm}$ (Figure $3 \mathrm{~b}$ ) suggesting that they are covered by 6MP molecules (see arrows in Figure 3c). The small corrugation value is consistent with molecular features dominated by electronic rather than topographic effects. The islands coexist with ordered molecular domains formed by stripes (Figure 3a) that also exhibit $h \approx$ 
$0.05 \mathrm{~nm}$ across them (Figure $3 \mathrm{~b}$ ). Note also that the stripes intersect steps at $45^{\circ}$, while two adjacent striped domains intersect at $90^{\circ}$ (Figure $3 \mathrm{c}$ ).
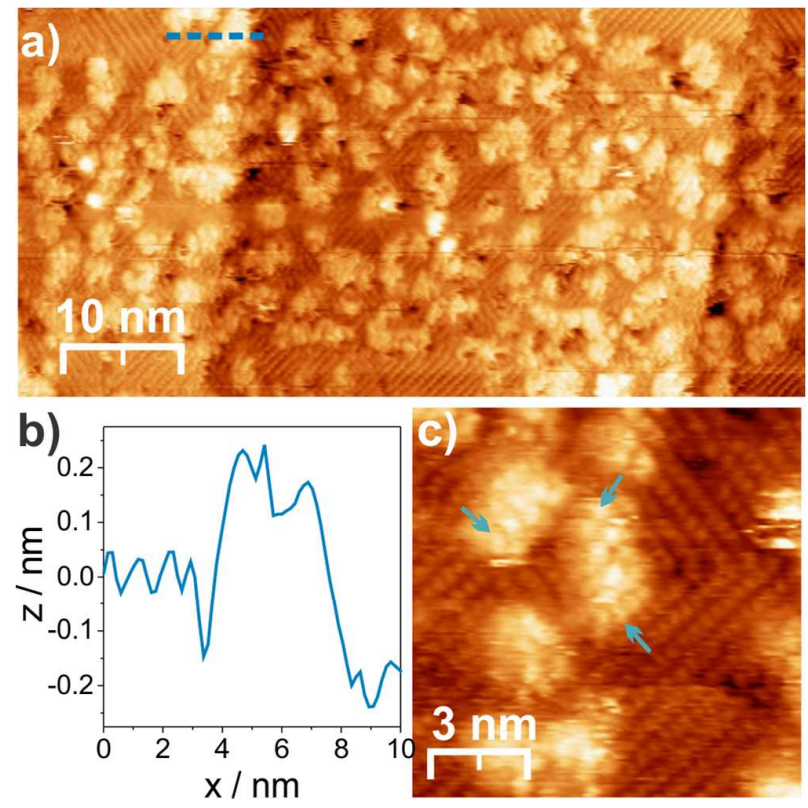

Figure 3 (a) Large scale STM image of 6MP on Au(001)-(hex) showing stripes and irregular $A u$ islands (b) cross-section along the blue dashed line in (a) showing the height of the islands, molecular features (left side and top of the island) and a step (right side) .(c) High resolution STM image of $6 M P$ on $A u(001)-(h e x)$ showing disordered molecules adsorbed on the islands (blue arrows).

The diffuse edges and irregular shape of these islands (Figure 3a,c) contrast the well-defined square or rectangular Au islands formed by electrochemical lifting of the hex reconstruction in the absence of aromatic thiol molecules. ${ }^{12}$ We recall that the lifting induced either by adsorption of molecules or by changing the electrochemical potential results in the expelling of $\mathrm{Au}_{\mathrm{ad}}$ in an equivalent amount to $\theta_{\mathrm{ad}}=0.25 \mathrm{ML}^{25}$ which either nucleate and grow into islands or reach step edges. It seems that the diffusion path of the $\mathrm{Au}_{\mathrm{ad}}$ is much smaller for the adsorption induced lifting presumably because the molecular lattice blocks the movement of the $\mathrm{Au}$ adatoms, thus leading to smaller and more irregular islands that are also covered by 6MP molecules (see arrows in Figure 3c). The estimation of the island coverage is difficult due to their irregular shape with disordered molecules at the boundaries, and also probably by convolution of the tip with the small islands, but a rough estimation yields about $\theta_{\text {isl }} \approx 0.3$, 
slightly larger than that expected considering that they are formed from the reconstruction $\left(\theta_{\mathrm{ad}}\right.$ $=0.25)$. This situation is completely different when $6 \mathrm{MP}$ adsorption takes place directly on the $\mathrm{Au}(100)-(1 \times 1)$ surface, where the molecules are not in contact with a large population of free $\mathrm{Au}$ adatoms. In this case the shape and size of the islands formed by electrochemical lifting remain unchanged after 6MP adsorption, i.e. the absorption process itself is not strong enough to mobilize a large amount of $\mathrm{Au}_{\mathrm{ad} .}{ }^{11}$

The STM image in Figure 4 was obtained in a particular region where there are practically no $\mathrm{Au}_{\mathrm{ad}}$ islands. Although areas like this are an exception we can still consider it to determine the molecular structure of the ordered domains coexisting with the islands. The image shows $\mathrm{Au}(001)$ terraces covered by rotated domains which were formed either by stripes where it is difficult to resolve individual molecules (red arrow in Figure 4a, phase A), or by stripes where individual molecules can be clearly distinguished (green arrow in Figure 4a, phase B). Note that there are no vacancy islands (depth=0.24 nm) on the terraces; instead we observe pinholes as dark holes corresponding to molecular vacancies (blue arrows in Figure 4a) with depth $<0.24 \mathrm{~nm}$. 

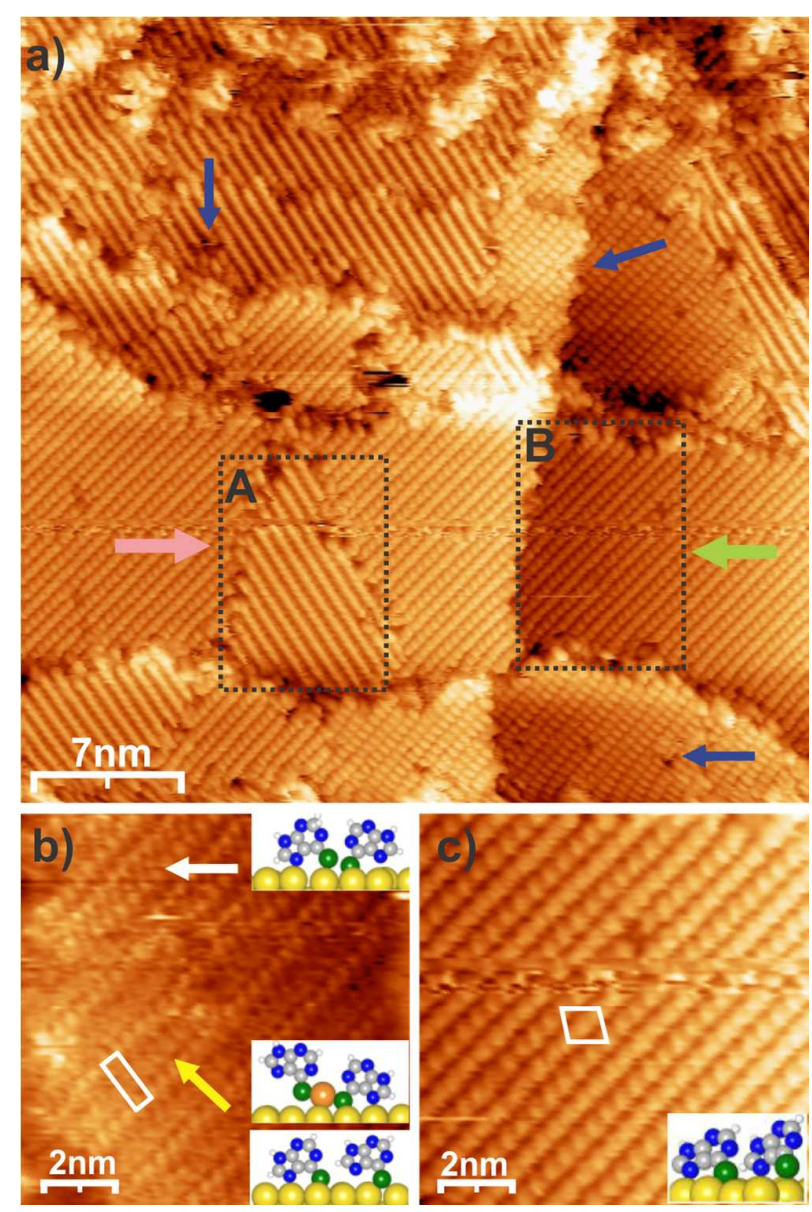

Figure 4. STM images of the 6MP-covered Au(001)-(hex) surface. (a) STM image (background subtraction) showing flat terraces and 6MP stripped phases: A domains (red arrow) and $B$ domains (green arrow). Steps and pinholes are also indicated (blue arrows). (b) High resolution STM image of type A domain. The head-to-tail (white arrow) and headto-head (yellow arrow) configurations, unit cell (white) and molecular arrangements from DFT models are also indicated. (c) High resolution STM image of type B domain. The unit cell (white) and the molecular arrangement from DFT model (taken from Ref 11) are indicated. Key: Au atoms (yellow), $A u_{a d}$ (orange), $S$ atoms (green), $C$ atoms (grey), $N$ atoms (blue), H atoms (white).

Figure $4 \mathrm{~b}$ shows the molecular structure of type A domains. At the upper left side of the image (white arrow) we can see bright rows separated by $0.65 \pm 0.03 \mathrm{~nm}$. In the remaining part of Figure $4 \mathrm{~b}$ one can see that one of the bright rows is absent so that they are separated by $\approx$ $1.3 \pm 0.05 \mathrm{~nm}$ (Figure 4b). Inside the rows we can detect individual spots separated by $0.42 \pm 0.03 \mathrm{~nm}$. Both structures can be related to the $(3 \sqrt{ } 2 \times \sqrt{ } 2) \mathrm{R} 45^{\circ}$ superstructure detected by LEED. The $0.65 \mathrm{~nm}$ dimension is close to the size of the $6 \mathrm{MP}$ molecule that has an elongated 
shape while the $\approx 0.4 \mathrm{~nm}$ distance inside the rows results from optimization of the $\pi-\pi$ interactions between adjacent aromatic rings. We propose head-to-tail (largely the most abundant) and head-to-head arrangements to explain the smaller $(0.65 \mathrm{~nm})$ and larger $(1.3$ $\mathrm{nm}$ ) separation between bright rows shown in Figure $4 \mathrm{~b}$. In fact, while the head-to-tail configuration was observed for this molecule on $\mathrm{Au}(100)-(1 \times 1)^{11}$ the head-to-head configuration has been frequently observed for different alkanethiols arranged parallel to the substrate. ${ }^{26-28}$. The larger size of the bright spots when the molecules are separated by $\approx 1.3$ $\mathrm{nm}$ suggests that they result from the convolution of the two adjacent $\mathrm{S}$ atoms, one from each molecule which, according to the literature, could form a disulfide bond ${ }^{26}$ or a staple unit by trapping one $\mathrm{Au}_{\mathrm{ad}}{ }^{29}$ (see schemes in Figure 4b-c). Therefore, both molecular arrangements exhibit the same surface coverage $\theta=0.33$ (see also Figure SI-1a).

On the other hand, type B domains consist of rows of bright spots that closely resemble the elongated shape of the 6MP molecule. The spots in neighboring rows are shifted forming quasi-rhomboidal structures with $0.5 \pm 0.03 \mathrm{~nm}$ and $0.7 \pm 0.03 \mathrm{~nm}$ intra and inter row distances, respectively (Figure $4 c)$. These structures can be described as a $(3 \times \sqrt{ } 10)$ lattice with $\theta=0.22$ (see Figure SI-1b) as already reported for $6 \mathrm{MP}$ on $\mathrm{Au}(001)-(1 \times 1)^{11}$, a fact that confirms the lifting of the hex reconstruction to form the $(1 \times 1)$ surface due to 6MP adsorption.

High resolution XPS has been used to explore the different chemical species present in the 6MP covered $\mathrm{Au}(001)$-(hex) surface. The $\mathrm{S} 2 \mathrm{p}$ spectrum (Figure SI-2a) reveals the presence of chemisorbed thiols ${ }^{6}$ without evidence of atomic sulfur or oxidized sulfur species, in agreement with previous data for this molecule on the $\mathrm{Au}(111)^{7}$ and $\mathrm{Au}(001)-(1 \times 1)$ surfaces. ${ }^{11}$ More important, the absence of the component corresponding to physisorbed thiols $($ at $\approx 163.5 \mathrm{eV}$ ) indicates that the disordered islands observed in the STM images are not aggregates of unbounded molecules. Also, the N 1s and C 1s XPS spectra (Figure SI$2 b, c)$ are similar to those reported for $6 \mathrm{MP}$ on the $\operatorname{Au}(001)-(1 \times 1)^{11}$ and $\operatorname{Au}(111)^{7}$ and 
consistent with the adsorption of intact dehydrogenated 6MP molecules, which are bound to the Au surface as thiolates.

We have performed reductive desorption curves in order to estimate the surface coverage of $6 \mathrm{MP}$ on the $\mathrm{Au}(001)$-hex surface. The curves show a main sharp peak located at a peak potential $E_{p}=-0.81 V$ (Figure SI-3) which appears at the same potentials as those found for $6 \mathrm{MP}$ on the $\mathrm{Au}(100)-(1 \times 1)^{11}$, which is another evidence for the lifting of the hex reconstruction upon 6MP adsorption. The charge density (q) involved in the peak for the $\mathrm{Au}(100)$ surface is $\mathrm{q}=47 \pm 7 \mu \mathrm{C} \mathrm{cm}^{-2}$ from which a thiol coverage $\theta=0.25$ is obtained. ${ }^{30-31} \mathrm{In}$ the present case it should be an average of the dense A and diluted B domains, as well as of the molecules adsorbed on the disordered Au island regions.

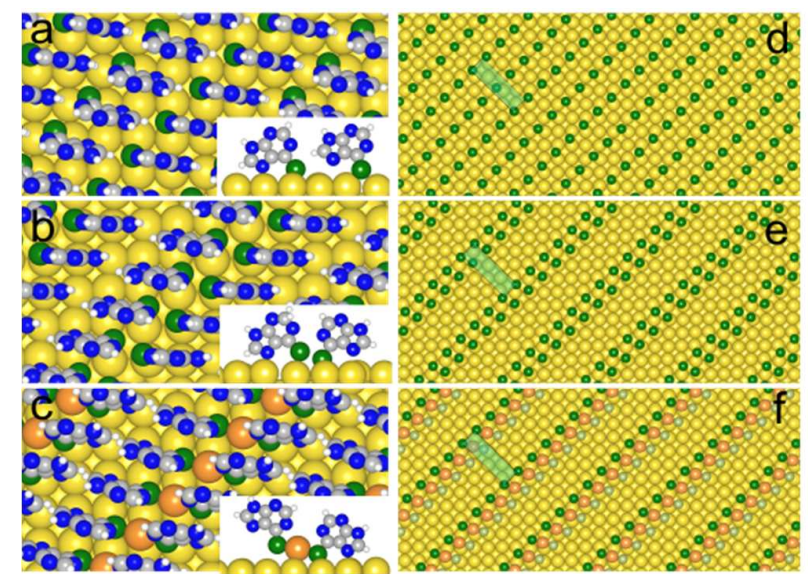

Figure 5. Optimized surface models on Au(001)-(1×1). (a, d): head-to-tail, $(b, e)$ : head-tohead, (c, f): head-Au ad head. (a-c) Top views. Insets: side views. Key: Au atoms (yellow), Au $u_{a d}$ (orange), $S$ atoms (green), $C$ atoms (grey), $N$ atoms (blue), $H$ atoms (white). (d-f) Positions of the $S$ heads (green) and Au adatoms (orange) for the different models. In (f) the disconnected $S$ atom is shown in light green. In (d-f) the unit cell is indicated in green.

DFT calculations were used to model the $(3 \sqrt{2} \times \sqrt{2}) \mathrm{R} 45^{\circ}$ surface structure containing two 6MP per unit cell in both head-to-tail (Figure $5 \mathrm{a}, \mathrm{d}$ ) and head-to-head configurations (Figure $5 \mathrm{~b}, \mathrm{e})$. In the head-to-tail configuration (Figure $5 \mathrm{a}, \mathrm{d}$ ) the $6 \mathrm{MP}$ molecules are bonded through their respective $\mathrm{S}$ atom at twofold bridge sites of the $\mathrm{Au}(001)$ surface, following the directions [110] and [1 $\overline{1} 0]$ and with the aromatic rings of both molecules oriented almost in 
the same direction. Besides, it can be observed that only one of the two 6MP (tilt angle $36^{\circ}$ ) is bound also on an Au atop site of the surface by the N(7) atom, while the $N(7)$ atom of the other 6MP (tilt angle $26^{\circ}$ ) is further away from the surface. Furthermore, we have simulated the head-to-head arrangements of the $(3 \sqrt{2} \times \sqrt{ } 2) \mathrm{R} 45^{\circ}$ lattice with two models whose main difference is the absence (Figure $5 \mathrm{~b}$, e) or presence of $\mathrm{Au}_{\mathrm{ad}}{ }^{29}$ (Figure 5c, f). In this case the adatom placed at a bridge site connects the $\mathrm{S}$ atoms of the two molecules, forming RS- $\mathrm{Au}_{\mathrm{ad}}{ }^{-}$ SR units.

On the other hand, we have assigned domain $B$ type to the more diluted $(3 \times \sqrt{ } 10)$ with $\theta=$ 0.22 that has been already proposed for $6 \mathrm{MP}$ molecules adsorbed on the $\mathrm{Au}(001)-(1 \times 1)$ after electrochemical lifting of the hex surface. ${ }^{11}$ The surface structure of domain B has been described by a rhomboidal $(3 \times \sqrt{ } 10)$ unit cell that consists of 6 MP molecules forming rows of rectangles $0.53 \times 0.73 \mathrm{~nm}^{2}$ in size rotated $\approx 45^{\circ}$ with respect to the substrate unit cell. Molecules are bonded through the $\mathrm{S}$ atoms which are placed at bridge and on top sites of the Au surface and by N(7) atoms at top sites. The tilt angle of the molecular plane with respect to the surface normal is $\alpha \approx 40^{\circ}$.

Now we will focus our analysis on the thermodynamic stability $(\gamma)$ of these models (see methods and Table 1- SI). The head-to-head configuration (Figure $5 \mathrm{~b}$, e) exhibits the lowest stability $\left(\gamma=-108.17 \mathrm{meV}^{-2}\right)$ due to its low adsorption energy $\left(E_{b}\right)$, which is largely improved by introducing the $\mathrm{Au}_{\mathrm{ad}}$ in the structure to form $\mathrm{RS}-\mathrm{Au}_{\mathrm{ad}}-\mathrm{SR}$ moieties (Figure $5 \mathrm{c}$ and $\mathrm{f}$ ). However, in this case we need an extra energy cost per 6MP to reconstruct the $1 \mathrm{x} 1$ surface and make $\mathrm{Au}_{\mathrm{ad}}$ available $\left(E_{\text {rec }}=+0.52 \mathrm{eV}\right)$. This energy cost markedly reduces the thermodynamic stability to $\gamma=-117.92 \mathrm{meV}^{-2}$, although it is still more stable than the adatom-free head-to-head configuration. Interestingly, we note that one of the S atoms of the staple initially bonded to the Au surface was, after optimization, spontaneously disconnected from the substrate (Figure 5f). On the other hand, the head-to-tail configuration (Figure 5a 
and d) exhibits the highest thermodynamic stability $\left(\gamma=-128.87 \mathrm{meVA}^{-2}\right)$ of the three models. Finally, the $(3 \times \sqrt{ } 10)$ lattice exhibits a similar binding energy $E_{b}=-3.24 \mathrm{eV}$ (equation [1] and SI- Table 1) but a much lower thermodynamic stability $\gamma=-83.3 \mathrm{meVA}^{-2}$ because of its lower molecular density.

The larger thermodynamic stability of the $(3 \sqrt{ } 2 \times \sqrt{ } 2)$ R $45^{\circ}$ lattices than that of the $(3 \times \sqrt{ } 10)$ lattice can be explained by the larger number of thiolate bonds ([equation 2]) and the optimization of intermolecular forces by $\pi-\pi$ stacking between the aromatic rings. Therefore, during self-assembly B domains should be slowly transformed into the more stable A domains as more 6MP molecules are adsorbed. In fact, Figure SI-1 c) shows that this process is possible by simple incorporation of additional 6MP molecules to the substrate, thus demonstrating the connection between the two surface structures. However, the coexistence of different phases in thiols SAMs at room temperature is a well-known phenomenon ${ }^{6}$ where molecules are kinetically trapped in metastable states, hindering the formation of denser phases. $^{29,32}$

Importantly the head-to-tail configuration (Figure $5 \mathrm{a}, \mathrm{d})$, the dominant $(3 \sqrt{2} \times \sqrt{2}) \mathrm{R} 45^{\circ} 6 \mathrm{MP}$ structure on $\mathrm{Au}(001)-(1 \times 1)$, is inconsistent with the presence of staples because the $0.4 \mathrm{~nm}$ distance is too small to accommodate these moieties, and because the molecules cannot maintain a parallel orientation due the $S$ atom hybridization. Also, for the $(3 \times \sqrt{ } 10)$ lattice the position and the orientation of the two 6MP molecules with their respective aromatic ring almost parallel do not allow the formation of the RS-Au$u_{a d}-\mathrm{SR}$ complex. In this case the $\mathrm{Au}_{\mathrm{ad}}$ should be bonded between both moieties through $\mathrm{S}-\mathrm{Au}$ bonds since the $\mathrm{S}$ atom is $\mathrm{sp}^{3}$ hybridized with bond angles of nearly $109.5^{\circ} .^{11}$

\section{Conclusions}

Our results for 6MP adsorption on the $\mathrm{Au}(001)$-(hex) surface reveal the low tendency of these aromatic thiol molecules to form ordered lattices containing staple moieties. The absence of 
staples was also observed for this molecule on $\mathrm{Au}(111)^{7}$ although in this case one could argue that adsorption was too weak to extract adatoms from terraces, as the lifting of the herringbone reconstruction only provides a negligible amount of adatoms $\left(\theta_{\mathrm{ad}}=0.05\right)$. The energy cost to extract adatoms from terraces could also explain the absence of staples when $6 \mathrm{MP}$ adsorption is performed directly on the $\mathrm{Au}(001)-(1 \times 1)$ surface. ${ }^{11}$ In contrast, for the $\mathrm{Au}(001)$-hex surface the lifting of the reconstruction provides a large excess of $\mathrm{Au}_{\mathrm{ad}}\left(\theta_{\mathrm{ad}}=\right.$ 0.25) so that this argument is not valid, i.e. there are always enough $\mathrm{Au}_{\mathrm{ad}}$ to form the staple moieties. However, even in this situation 6MP molecules organize to yield a bridging configuration while the ejected $\mathrm{Au}_{\mathrm{ad}}$ are trapped inside the ordered lattices forming irregular $\mathrm{Au}$ islands. Therefore, our investigation reveals that the formation of thiol-gold adatom complexes is not always a thermodynamically favored process and depends strongly on the nature of the thiol molecule. To sum up, we present a new scenario in the surface chemistry of the thiol-gold system: a correct selection of the thiol molecule allows us to tailor the structure of the thiol-Au interface in SAMs, which opens the possibility for applications where tailor-made interfaces are relevant.

\section{Supporting Information}

Supporting information contains graphical explanation of phase transition from domain B to domain A and their units cells, XPS plots, electrochemical reductive desorption of 6MP and table containing the energetic and structural data for 6MP surface structure on $\mathrm{Au}(001)$-hex obtained by DFT.

\section{Acknowledgements}

The authors acknowledge financial support from ANPCyT (PICT 2012-0836) and CONICET (PIP 0093). P.C. acknowledges MINECO (ENE2016-74889-C4-2-R, AEI-FEDER-UE). F. L. 
M. acknowledges a doctoral fellowship from CONICET and a Max Planck Stipendium in

Prof. Kern's group.

\section{References}

(1) Hakkinen, H. The Gold-Sulfur Interface at the Nanoscale. Nat. Chem. 2012, 4, 443-455.

(2) Pensa, E.; Cortés, E.; Corthey, G.; Carro, P.; Vericat, C.; Fonticelli, M. H.; Benítez, G.; Rubert, A. A.; Salvarezza, R. C. The Chemistry of the Sulfur-Gold Interface: In Search of a Unified Model. Acc. Chem. Res. 2012, 45, 1183-1192.

(3) Jadzinsky, P. D.; Calero, G.; Ackerson, C. J.; Bushnell, D. A.; Kornberg, R. D. Structure of a Thiol Monolayer-Protected Gold Nanoparticle at $1.1 \AA$ Resolution. Science 2007, 318, 430-433.

(4) Cossaro, A.; Mazzarello, R.; Rousseau, R.; Casalis, L.; Verdini, A.; Kohlmeyer, A.; Floreano, L.; Scandolo, S.; Morgante, A.; Klein, M. L., et al. X-Ray Diffraction and Computation Yield the Structure of Alkanethiols on Gold(111). Science 2008, 321, 943-946.

(5) Reimers, J. R.; Ford, M. J.; Marcuccio, S. M.; Ulstrup, J.; Hush, N. S. Competition of Van Der Waals and Chemical Forces on Gold-Sulfur Surfaces and Nanoparticles. Nat. Rev. Chem. 2017, 1, 0017.

(6) Vericat, C.; Vela, M. E.; Benitez, G.; Carro, P.; Salvarezza, R. C. Self-Assembled Monolayers of Thiols and Dithiols on Gold: New Challenges for a Well-Known System. Chem. Soc. Rev. 2010, 39, 1805-1834.

(7) Pensa, E.; Carro, P.; Rubert, A. A.; Benítez, G.; Vericat, C.; Salvarezza, R. C. Thiol with an Unusual Adsorption-Desorption Behavior: 6-Mercaptopurine on Au(111). Langmuir 2010, 26, 17068-17074.

(8) Jin, Q.; Rodriguez, J. A.; Li, C. Z.; Darici, Y.; Tao, N. J. Self-Assembly of Aromatic Thiols on $\mathrm{Au}(111)$. Surf. Sci 1999, 425, 101-111.

(9) Pensa, E.; Rubert, A. A.; Benitez, G.; Carro, P.; Orive, A. G.; Creus, A. H.; Salvarezza, R. C.; Vericat, C. Are 4-Mercaptobenzoic Acid Self Assembled Monolayers on Au(111) a Suitable System to Test Adatom Models? J. Phys. Chem. C 2012, 116, 25765-25771.

(10) Parker, W. B. Enzymology of Purine and Pyrimidine Antimetabolites Used in the Treatment of Cancer. Chem. Soc. Rev. 2009, 109, 2880-2893.

(11) Lobo Maza, F.; Grumelli, D.; Carro, P.; Vericat, C.; Kern, K.; Salvarezza, R. C. The Role of the Crystalline Face in the Ordering of 6-Mercaptopurine Self-Assembled Monolayers on Gold. Nanoscale 2016, 8, 17231-17240.

(12) Grumelli, D.; Cristina, L. J.; Maza, F. L.; Carro, P.; Ferrón, J.; Kern, K.; Salvarezza, R. C. Thiol Adsorption on the Au(100)-Hex and Au(100)-(1 × 1) Surfaces. J. Phys. Chem. C 2015, $119,14248-14254$.

(13) Horcas, I.; Fernandez, R.; Gomez-Rodriguez, J. M.; Colchero, J.; Gomez-Herrero, J.; Baro, A. M. Wsxm: A Software for Scanning Probe Microscopy and a Tool for Nanotechnology. Rev. Sci. Instrum. 2007, 78, 013705-8.

(14) Yeh, J. J.; Lindau, I. Atomic Subshell Photoionization Cross Sections and Asymmetry Parameters: $1 \leqslant Z \leqslant 103$. Atomic Data and Nuclear Data Tables 1985, 32, 1-155.

(15)Hermann, K.; Van Hove, M. A. Http://Www.Fhi-Berlin.Mpg.De/Khsoftware/Leedpat/Leedpat4 Leed Pattern Analyzer.

(16) Kresse, G.; Furthmüller, J. Efficiency of Ab-Initio Total Energy Calculations for Metals and Semiconductors Using a Plane-Wave Basis Set. Comput. Mater. Sci. 1996, 6, 15-50.

(17) Kresse, G.; Hafner, J. Ab Initio. Phys. Rev. B 1993, 48, 13115-13118.

(18) Dion, M.; Rydberg, H.; Schröder, E.; Langreth, D. C.; Lundqvist, B. I. Van Der Waals Density Functional for General Geometries. Phys. Rev. Lett. 2004, 92, 246401.

(19) K, K.; Bowler, D., R. ; Michaelides, A. Chemical Accuracy for the Van Der Waals Density Functional. J. Phys. Condens. Matter 2010, 22, 022201.

(20) Blöchl, P. E. Projector Augmented-Wave Method. Phys. Rev. B 1994, 50, 17953-17979.

(21) Monkhorst, H. J.; Pack, J. D. Special Points for Brillouin-Zone Integrations. Phys. Rev. B 1976, 13, 5188-5192.

(22) Reimers, J. R.; Ford, M. J.; Halder, A.; Ulstrup, J.; Hush, N. S. Gold Surfaces and Nanoparticles Are Protected by $\mathrm{Au}(0)-$ Thiyl Species and Are Destroyed When Au(I)-Thiolates Form. Proc. Natl. Acad. Sci. 2016, 113, E1424-E1433.

(23) Pearson, W. B. In Handbook of Lattice Spacing and Structure of Metals; Pergamon Press, Inc.: New York, 1958. 
(24) Jiang, Y.; Liang, X.; Ren, S.; Chen, C.-L.; Fan, L.-J.; Yang, Y.-W.; Tang , J.-M.; Luh, D.-A. The Growth of Sulfur Adlayers on Au(100). J. Chem. Phys. 2015, 142, 064708 1-11.

(25) Hölzle, M. H.; Wandlowski, T.; Kolb, D. M. Phase Transition in Uracil Adlayers on Electrochemically Prepared Island-Free Au(100)-(1 × 1). J. Electroanal. Chem. 1995, 394, $271-$ 275.

(26) Schreiber, F. Structure and Growth of Self-Assembling Monolayers. Prog. Surf. Sci. 2000, $65,151-257$.

(27) Grumelli, D.; Maza, F. L.; Kern, K.; Salvarezza, R. C.; Carro, P. Surface Structure and Chemistry of Alkanethiols on Au(100)-(1 × 1) Substrates. J. Phys. Chem. C 2016, 120, 291-296.

(28) Eisuke, I.; Takayuki, A.; Masahiko, H.; Jaegeun, N. Surface Potential Change Depending on Molecular Orientation of Hexadecanethiol Self-Assembled Monolayers on Au(111). Bull. Korean Chem. Soc. 2009, 30, 1309-1312.

(29) Löfgren, J.; Grönbeck, H.; Moth-Poulsen, K.; Erhart, P. Understanding the Phase Diagram of Self-Assembled Monolayers of Alkanethiolates on Gold. J. Phys. Chem. C 2016, 120, 12059 12067.

(30) Reyes, E.; Madueño, R.; Blázquez, M.; Pineda, T. Facile Exchange of Ligands on the 6Mercaptopurine-Monolayer Protected Gold Clusters Surface. J. Phys. Chem. C 2010, 114, 15955-15962.

(31) Madueño, R.; Sevilla, J. M.; Pineda, T.; Román, A. J.; Blázquez, M. A Voltammetric Study of 6-Mercaptopurine Monolayers on Polycrystalline Gold Electrodes. J. Electroanal. Chem. 2001, $506,92-98$.

(32) Leung, T. Y. B.; Gerstenberg, M. C.; Lavrich, D. J.; Scoles, G.; Schreiber, F.; Poirier, G. E. 1,6-Hexanedithiol Monolayers on Au(111): A Multitechnique Structural Study. Langmuir 2000, $16,549-561$.

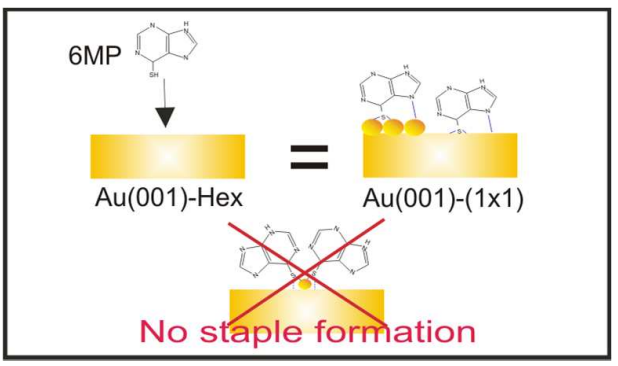

